\title{
Case of Acute Strangulation of the Uterus by Torsion of the Body on the Cervix: Supra-cervical Hysterectomy : Recovery.
}

\author{
Bу John D. Malcolm, F.R.C.S. (Edin.). \\ Consulting Surgeon, The Samarilan Free Hospital.
}

Rotatron of the whole uterus with an ovarian or uterine tumour is not very uncommon, the displacement being at most something over half a revolution. I have never seen complete arrest of the blood supply from this cause, and I have not before seen a case like the following :-

On November 3 , I915, a rather frail lady, æt. 70, married, but never pregnant, complained after dinner of pelvic pain. Next morning she was better and took her breakfast in bed as usual; but she could not get up afterwards because the pain became intense and was accompanied by vomiting. Towards evening the temperature rose to $\operatorname{IoI}^{\circ} \mathrm{F}$. I saw her late that night with Dr. Hewetson and Dr. Watson, of Reigate, and found a hard, nearly round pelvic tumour, median in position and about four inches in diameter. Behind this, and also in the middle line, lay an elongated, irregularly-oval mass measuring apparently about $3 \frac{1}{2}$ inches from above downwards, by $I \frac{1}{2}$ inches from side to side. This was fairly soft, but of uneven consistence. It seemed to be attached to the hard mass in front, and there was a small, soft, short cervix uteri below. The rectum was firmly compressed, but the abnormal parts, which were very tender, seemed to be slightly moveable as one mass in the pelvis. It was known that a fibromyoma existed before the patient's menstruation ceased, and it was easy to surmise that something had twisted, but the condition behind the hard tumour puzzled us all.

It was decided to open the abdomen at once, and on doing so a fibromyoma was exposed. When this was raised out of the pelvis by folding it backwards the condition shown diagramatically in Fig. I was discovered. There were no adhesions anywhere, but the fibromyoma and the body of the uterus were of a deep blue-black colour from congestion caused by a complete revolution of these parts on the cervix uteri. The left tube and ovary were above the site of the torsion, the right ovary was below, and the right tube was involved in it. Although the operation was performed only sixteen hours after the pain became severe and about twenty-eight hours from the first onset, the condition caused a distinctly un- 
pleasant odour. The parts drawn in Fig. 2 were removed by dividing the uterus at the junction of the congested with the healthy tissues and securing the vessels which were few and small. The cut peritoneal edges were sewn together and when released the stump fell well down in the pelvis.

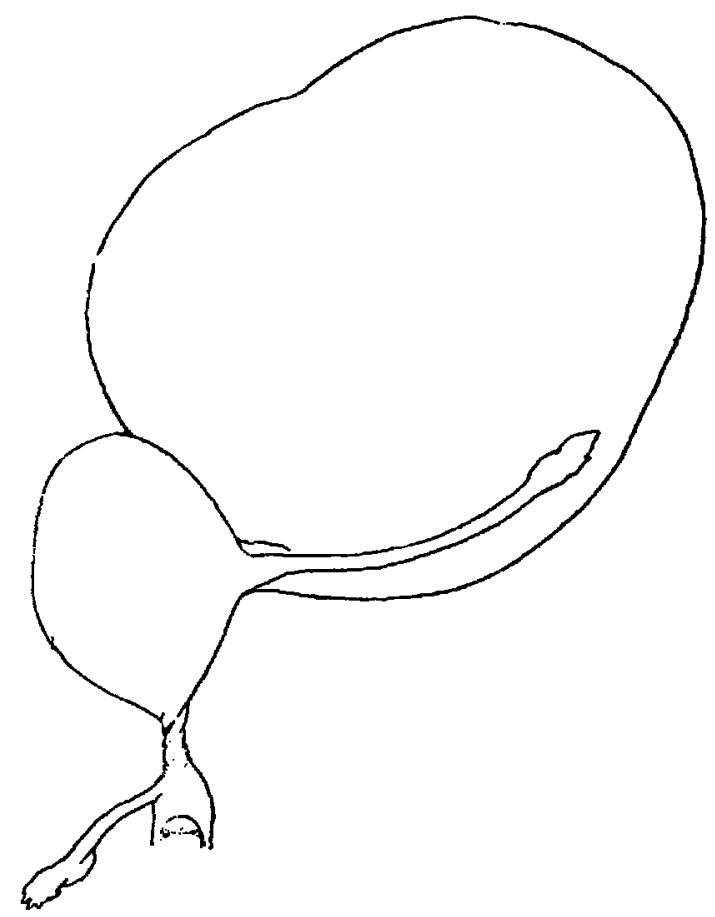

Fig I.-Showing diagrammatically the relative proportions of the congested body and the senile cervix uteri, the tightness of the structure, and the portion of the ovaries.

This patient had always been slow in recovering from illness, and after the operation there was considerable difficulty and anxiety on account of an inactive state of the bowels accompanied by flatulent distension, but by a judicious use of intestinal stimulants recovery was gradually established. In the temporary absence of Dr. Hewetson, Dr. Watson guided the patient through the most critical stage, and he found pituitary extract definitely useful as a promoter of peristalsis in this case.

The uterine body, as shown in Fig. 2, was as big as that of a woman in the child-bearing period, but its size was due to an intense congestion. The cut surface, as drawn, has a wide area which does not suggest a constriction, but the parts were untwisted before they were divided and the removal was effected so as to take a wedge out of the part left, the result being a nearly flat surface. 
There can be no doubt that before the torsion occurred the uterus was very soft from senility and stretched and narrowed above the vagina by traction of the tumour. It would hardly seem possible that the torsion found could exist without these conditions.

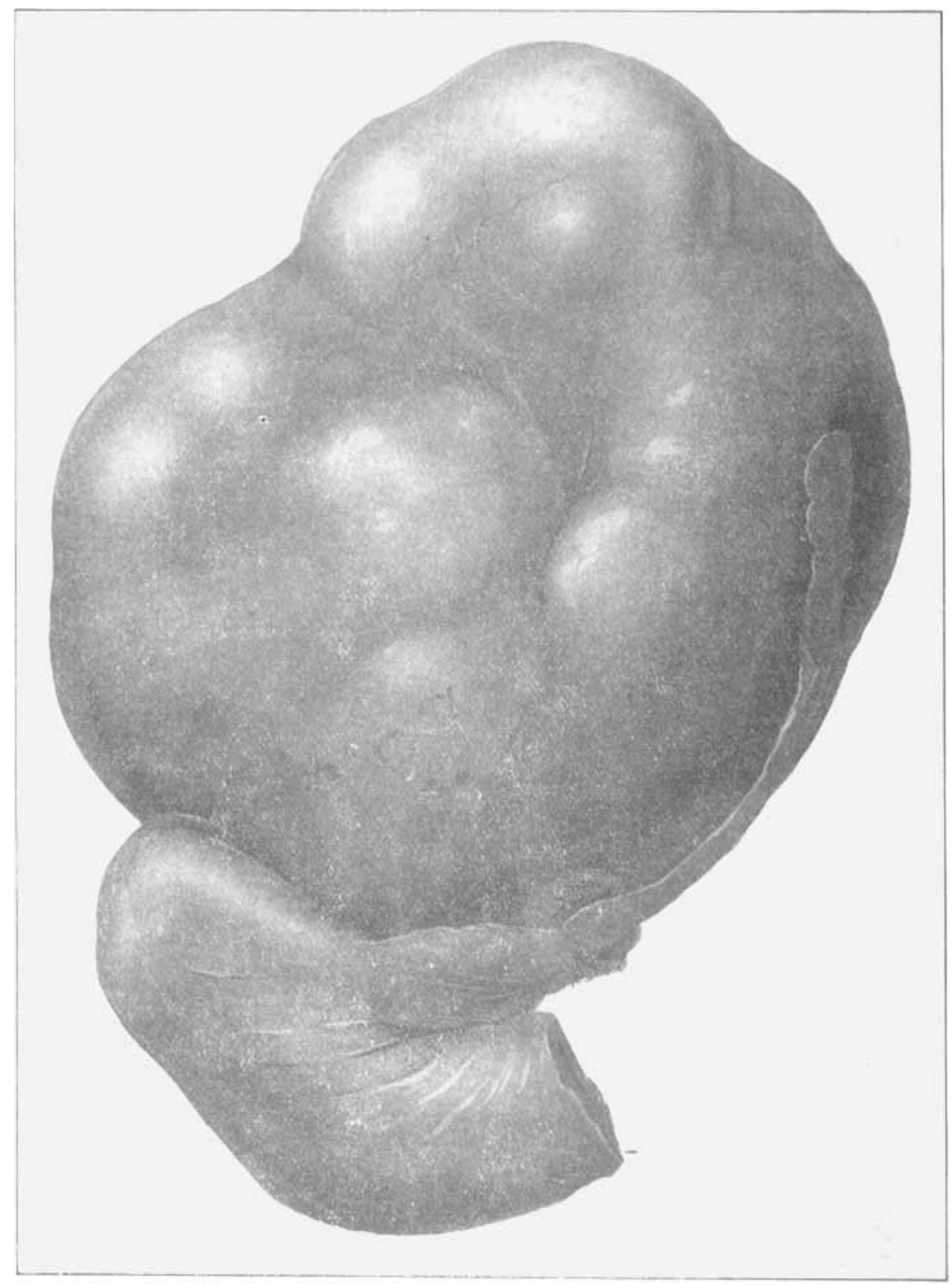

Fig. 2.- The uterus, tumour, and left ovary. 